\title{
Formación inicial docente y espacios fronterizos. Profesores en aulas culturalmente diversas. Región de Arica y Parinacota ${ }^{1}$
}

\author{
Initial teacher training and border areas. \\ Teachers in culturally diverse classrooms. Arica and Parinacota region
}

\author{
Eugenio Sánchez Espinoza ${ }^{a}$, Carmen Norambuena Carrasco $^{b}$ \\ ${ }^{a}$ Facultad de Ciencias Humanas. Universidad Arturo Prat, Chile. \\ eusanchez@unap.cl \\ ${ }^{b}$ Departamento de Historia. Universidad de Santiago de Chile. \\ carmen.norambuena@usach.cl
}

\section{RESUMEN}

El artículo tiene por objetivo el análisis de la formación inicial de los docentes en ejercicio insertos en un espacio educativo fronterizo y culturalmente diverso y, a partir de este, determinar si las prácticas educativas de los docentes de enseñanza básica de las escuelas públicas de la región de Arica y Parinacota pueden ser consideradas interculturales. El trabajo posee un enfoque cualitativo que se inició con la entrevista de informantes claves (profesores en ejercicio). Los resultados obtenidos a partir del procesamiento cualitativo de las entrevistas de profesores en ejercicio dejan en evidencia la ausencia de la temática intercultural, teórica y práctica durante la formación inicial; lo que se traduce en prácticas surgidas desde la experiencia e iniciativa personal de cada profesor, más que desde de un trabajo sistemático para su desempeño en contextos de diversidad cultural.
\end{abstract}

Palabras clave: diversidad cultural, formación inicial docente, región fronteriza.

\section{ABSTRACT}

The objective of the article is to analyze the initial training of practicing teachers in a frontier region and culturally diverse educational space and, based on this, to determine whether the educational practices of teachers of primary education in public schools in the region of Arica and Parinacota can be considered intercultural. The work has a qualitative approach that started with the interview of key informants (practicing teachers). The results obtained from the qualitative processing of the interviews of practicing teachers shows the absence of the intercultural, theoretical and practical theme, during the initial training which translates into practices arising from the experience and personal initiative of each teacher, more than from a systematic work for their performance in contexts of cultural diversity.

Key words: cultural diversity, initial teacher training, frontier region.

\footnotetext{
Los autores agradecen el financiamiento recibido a través del Proyecto de Investigación FONDECYT N 1191317.
} 
Estudios Pedagógicos XLV, $\mathrm{N}^{\circ}$ 2: 83-99, 2019

FORMACIÓN INICIAL DOCENTE Y ESPACIOS FRONTERIZOS. PROFESORES EN AULAS CULTURALMENTE DIVERSAS. REGIÓN DE ARICA Y PARINACOTA

\section{INTRODUCCIÓN}

Desde la década de los noventa del siglo pasado la globalización ha transformado a Chile en una sociedad receptora de migrantes de diversas nacionalidades que traen consigo sus propios modos de vida y prácticas culturales (Pries, 1999a y 1999b). Si bien es cierto que esta realidad se presenta a nivel nacional, no es menos cierto que los espacios fronterizos fueron lugares propicios para el desarrollo de una diversidad cultural que acepta los valores y estilos de vida de las personas, no obstante, sus diferencias (Becerra, 2005) tal cual ocurre en el extremo norte de Chile.

La Región de Arica y Parinacota, como territorio de frontera, acogió a un gran número de extranjeros migrantes que, al sumarse a las comunidades étnicas de la región, constituyó un espacio habitado por una población con características demográficas particulares con un dinamismo cultural integrado y formado a través de un tejido de relaciones transfronterizas a distinto nivel (Becerra, 2005; Guizardi y Garcés, 2012; Norambuena, 2002; 2004; Tapia, 2015). Esta realidad determinada por el proceso migratorio y una alta presencia de población perteneciente al pueblo aymara, y la consecuente generación de espacios culturalmente diversos en las zonas fronterizas, representa un escenario que se traslada a las aulas de la región. Las salas de clases albergan a un alumnado perteneciente a diversas minorías, étnicas y extranjera, que plantean nuevas demandas a la educación chilena, en su conjunto, y en forma más específica a los docentes en ejercicio y sus prácticas pedagógicas que deben atender con una cultura antidiscriminatoria, más integradora que excluyente, a estos grupos que forman el estudiantado cada vez más heterogéneo en las aulas de todo nuestro país (Barrios y Palou, 2014; Stefoni, Stang y Riedemann, 2016).

Los otrora espacios educativos, relativamente homogéneos, han dado paso a aulas diversas debido a la llegada de estudiantes migrantes, lo que ha implicado para los docentes y establecimientos educacionales importantes desafíos (Sanhueza, Friz, y Quintriqueo, 2014). Estos desafíos no solo se relacionan con cuestionar las percepciones que los docentes tienen sobre la migración, sino que también a replantearse las estrategias y actividades en la sala de clases y en su institución (Celedón, Naudon y Carmona, 2018).

Este artículo presenta parte de los resultados de una investigación sobre estos cambios culturales y, más precisamente, analiza la Formación Inicial Docente (FID) recibida por los profesores que se desempeñan en las aulas de escuelas públicas de enseñanza básica culturalmente diversas de la región fronteriza de Arica y Parinacota.

\section{CONTEXTO EDUCACIONAL Y LA EDUCACIÓN MULTICULTURAL}

A nivel nacional, se podría decir que actualmente estamos en presencia de una multiculturalidad que se vive en muchas salas de clases del país (Stefoni et al., 2016). En el caso específico de la región fronteriza de Arica y Parinacota esta realidad se caracteriza porque en las escuelas básicas existe una alta presencia de niños pertenecientes al pueblo aymara e hijos de migrantes de diversas nacionalidades (Joiko y Vásquez, 2016; Mondaca y Gajardo, 2015; Mondaca, Gajardo y Sánchez 2016; Mondaca, Rivera, y Gajardo, 2014). Estos estudiantes se concentran mayormente en las escuelas públicas de la comuna de Arica (Departamento Administración Educación Municipal Arica, 2017). 
Estos espacios educativos son complejos desde el punto de vista cultural, puesto que representan espacios multiculturales en los cuales los grupos de extranjeros y los grupos étnicos superponen relaciones y desafían los procesos identitarios. Asimismo, en este relativamente nuevo contexto, los profesores deben estar más dispuestos a aceptar la realidad y a desarrollar competencias para enfrentarla y adaptarse (Cerón, Pérez y Poblete, 2017). El docente, como agente clave en el proceso de enseñanza y aprendizaje de sus estudiantes, debe comprender y asumir que dicho proceso se complejiza en un ambiente de diversidad cultural, lo que se incrementa cuando la FID recibida está más bien dirigida y pensada para un estudiantado homogéneo y no el existente en las aulas fronterizas de la Región de Arica y Parinacota.

El desarrollo de competencias pertinentes para estos contextos escolares es vital cuando se considera el proceso de enseñanza-aprendizaje en su conjunto. En este proceso intervienen tanto el trabajo en la sala de clases como la comunicación y la manera en la que se abordan las problemáticas educativas que puedan surgir desde la diversidad cultural que en definitiva definen contextos precisos para hacer posible el aprendizaje. Si este clima de microsociedad es bueno, los aprendizajes también lo serán.

El desarrollo de habilidades o competencias específicas necesarias para abordar y desempeñarse en establecimientos educativos y salas de clases con diversidad cultural o multiculturales en un contexto formal de educación, hace que los profesores que ahí trabajan muchas veces descubran que su FID no los preparó de la forma más idónea para estos contextos (Alliaud y Vezub, 2014; Becerra, 2005; Valenzuela, Muñoz y Marfull, 2018). En este sentido, un profesor que se desempeña en un aula que alberga, por ejemplo, un $80 \%$ de estudiantes de otra nacionalidad y de ascendencia aymara en una escuela pública de la comuna de Arica, debiera poseer conocimientos teóricos y prácticos relativos a la educación en y para la diversidad cultural, y manejar el enfoque educativo intercultural para abordarla.

El análisis de las vivencias, del ejercicio y práctica docente en estos contextos es necesario para trazar estrategias que permitan a los profesionales de la educación el reconocimiento del otro en su diversidad, lo que se debe cultivar como una actitud propicia para el desarrollo de un diálogo en el que cada cultura interviniente se despliegue en todas sus posibilidades de significación y de comunicación. De esta manera, el profesor logra el desarrollo de valores universales, tolerancia y respeto por el otro diferente al interior de la escuela pues, de lo contrario, la educación formal perdería su sentido y la escuela, como institución, dejaría de ser efectiva como uno de los elementos que ayuda a la mantención del tejido social (Ruffinelli, 2016; Zapata 2013, 2014). Por otra parte, el contexto del espacio fronterizo obliga a indagar la forma en que conceptos como el de educación intercultural, interculturalidad y multiculturalidad fueron tratados durante la FID con el fin de que ellos se traduzcan en una forma de acercarse y aprehender la realidad cultural existente en el contexto educativo.

El enfoque intercultural, como mirada teórica, nos permite pensar en la diversidad humana y en formular vías alternativas de acción y pensamiento en educación (Aguado, 2009; Dietz, 2003, 2013). Por consiguiente, un enfoque de educación intercultural supone reconocer por parte de los actores su carácter práctico y hermenéutico al unísono, toda vez que nos permite internalizar las diferencias y trabajar con ellas en el espacio educativo (Aguado, 2009). Al imponerse en la práctica docente este enfoque de educación intercultural obliga al profesor a determinadas formas de hablar y de actuar en situaciones educativas 
específicas (Aguado, 2009) que se dan al interior de los establecimientos educativos en contextos multiculturales fronterizos.

La aplicación de la educación intercultural, que involucra una perspectiva intercultural del tejido social, conlleva un proceso reflexivo de parte del docente para no detenerse en el referente cultural como determinante de comportamientos, sino sobre la manera en que la persona emplea los rasgos culturales para decir y decirse, lo que se traduce en pensar desde la condición de que somos iguales en términos de dignidad para dejar de utilizar la “diversidad" como pretexto de una posible exclusión social (Morales, 2015). En este sentido, cobra relevancia el proceso de enseñanza de una cultura diversa, ya que el educador debe asegurarse de poseer un conocimiento claro de las reglas y significados que subyacen en el proceso de construcción e interpretación de un mundo particular y sus formas de construir realidades intersubjetivas (Geeregat, Vásquez y Fierro, 2012) cuando, por ejemplo, se trata aspectos relativos a las ritualidades del pueblo aymara. De esta forma, los contextos y escenarios fronterizos, en el ámbito educativo, deben ser un catalizador que amerita una formación universitaria que contemple una perspectiva de educación intercultural a fin de lograr la inclusión de todos los grupos presentes en la escuela y salas de clases de forma transversal. Este argumento permitirá que los futuros profesores desarrollen iniciativas educativas orientadas a transformar la escuela en un verdadero espacio de aprendizaje para la tolerancia y respeto de las diferencias y características culturales.

Las Instituciones de Educación Superior (IES) no solo deben entregar una visión general de la realidad local y de las características culturales de los sujetos o grupos que habitan en la región. Geeregat et al., (2012) manifiestan que en los espacios fronterizos el currículo de las IES se tensiona al asumir procesos de formación de actores educativos relevantes en contextos inter y multiculturales, es decir, qué contenidos y de qué forma deben ser trabajados con los estudiantes, por ejemplo. Puntualizando, la formación de estudiantes de pedagogía con competencias de diferente orden debe formar parte del perfil profesional integral y como desafío institucional, así la escuela cumple su rol de agencia que permite la difusión de valores culturales y la reproducción de los principios de tolerancia y convivencia al interior de la nación, además de una formación ciudadana que no solo se fortalece en y con los ritos cívicos (Díaz, 2003).

\section{FORMACIÓN INICIAL Y EDUCACIÓN INTERCULTURAL}

El proceso de formación inicial conlleva problemáticas y situaciones particulares que dependen del contexto donde se desarrolla; pero el contexto cambia rápidamente y el proceso formativo pareciera que lo hace a un ritmo más lento, con lo cual adquiere siempre un carácter desactualizado y desconectado de la realidad educativa (Vaillant, 2013; Vezub, 2016). La formación inicial debe ser asumida como una etapa durante la cual se desenvuelve una práctica educativa intencional, sistemática y organizada, destinada a preparar a los futuros profesores para desempeñarse en su función (Enríquez, 2007) y en la que se promueve la internalización de conocimientos teóricos y prácticos que los habiliten para ejercer su práctica profesional. Asimismo, la formación docente es un término más global que hace referencia al desarrollo profesional, visto como un proceso evolutivo continuo, en el que los profesores aprenden a enseñar y mejorar su repertorio de capacidades profesionales, individuales y colectivas, en el contexto de sus instituciones, lo que permite 
superar la tradicional yuxtaposición entre formación inicial y perfeccionamiento docente (Hirmas y Cortés, 2013).

Para Enríquez (2007) la formación inicial es una etapa preparatoria que abarca un período definido y relativamente corto que habilita a un sujeto determinado a ejercer una profesión. Además, involucra una práctica educativa que se desarrolla en un contexto socio-político determinado que implica aspectos sociales, políticos y culturales. También conlleva una práctica intencional destinada a proporcionar a los docentes en formación ciertos conocimientos conceptuales, actitudinales y procedimentales que les servirán de referencia para trabajar en la escuela; junto a una práctica sistemática y organizada de carácter formativo. Vale decir, que las acciones que se ponen en juego no son improvisadas, sino por el contrario, cada uno de los elementos está relativamente organizado y las fases están definidas con antelación. La FID es, por tanto, un espacio cuyos destinatarios constituyen un grupo de docentes en formación, dispuesto y comprometido en su proceso formativo; es, además, un espacio destinado a la formación para un puesto de trabajo. Finalmente, esta formación les otorga a los sujetos una acreditación reconocida socialmente que los habilita a ejercer una práctica profesional (Enríquez, 2007). Por consiguiente, la FID es una práctica educativa multidimensional y pluriparadigmática que involucra una red de dimensiones políticas, sociales, culturales y educativas que hacen de este proceso un complejo que se encuentra abierto al análisis y multiplicidad de perspectivas. De esta forma la FID no puede considerarse una actividad aislada e independiente del contexto en el que se desarrolla. Es por esto por lo que el docente debe ser un sujeto en constante reflexión del escenario en que se desempeña y de la realidad sociocultural en que se encuentran insertos sus estudiantes.

Estos procesos de formación a los que han sido sometidas generaciones de profesores hoy evidencian carencias en la adaptación a las nuevas realidades y contextos, y así se llegan a identificar necesidades formativas en el conocimiento de aquellos aspectos del desempeño profesional en los que el profesorado presenta insuficiencias o considera relevantes para acometer su labor diaria (Dorfsman, 2012).

La atención educativa en y para la diversidad cultural de la zona norte de nuestro país implica desarrollar un análisis de cómo se construye la diferencia en estos espacios, pero sin perder de vista los múltiples procesos de construcción de la identidad de los grupos que habitan la Región de Arica y Parinacota; junto a las políticas educativas, los programas universitarios y el rol de la educación superior en el desarrollo de las zonas fronterizas. Las IES tienen el deber de entregar a sus estudiantes, que se desenvolverán en el campo de la educación, un conjunto de estrategias de trabajo relacionadas con la organización, la planificación y la metodología (García, 2005) para que, capacitados en un amplio repertorio pedagógico, desarrollen una tarea tan relevante como la de enseñar en un espacio multicultural fronterizo a la vez que inician un proceso de crecimiento profesional que los transforma en individuos llanos al reto y oportunidad que representa la diversidad.

Cabe entender que un proceso como la FID con su objetivo de formación tan relevante en interculturalidad, no se puede lograr, solamente, abarrotando mallas curriculares con más contenidos, sino que es necesario aportar nuevos enfoques formativos que contemplen la previsible heterogeneidad de los estudiantes a los que harán frente en las aulas (Essomba, 2008), sobre todo en una región fronteriza con las características particulares de la Región de Arica y Parinacota. Esto nos lleva a que el estudiante de pedagogía en formación logre adquirir y desarrollar auténticas competencias interculturales, ya que estas 
parecen ser esenciales para un profesional de la educación que se desenvuelva en la actual sociedad abiertamente diversa y democrática. Dicho de otro modo, se pone de manifiesto la necesidad de poseer, en el campo de la FID, una preparación que facilite el desarrollo y la consolidación de capacidades de orden intercultural en los estudiantes universitarios (García, 2005). La educación intercultural no solo es, ni debe ser, un contenido más sino un enfoque educativo para internalizar y aprehender el mundo y no un mero discurso en manos del profesor. Así, la reflexión en educación intercultural implicaría una preocupación fundamental: la de imaginar una pedagogía apropiada a las sociedades multiculturales en la cual el diálogo se construye sobre la base del respeto de la diversidad cultural y nos permite desarrollar una percepción del mundo, como un lugar histórico a compartir entre todos los seres vivientes (Marín, 2009).

La FID junto al desarrollo de competencias interculturales o la formación docente en educación intercultural y a la vez a la formación capacitada del docente para estos contextos es una transformación profunda en el sujeto, una forma distintiva de ver el mundo y experimentar la vida con quienes les rodean (alumnos, apoderados, colegas, etc.), que implica respetar y reconocer la existencia de la alteridad que, en definitiva, nos obliga a reflexionar e interrogarnos sobre la calidad de nuestras relaciones con los demás (Marín, 2009). Esta situación hace que la escuela adquiera centralidad como una institución mediadora entre el resto (marginados y excluidos al ser extranjeros, indígenas, pobres, etc.) y la sociedad, ya que es la llamada a cumplir el rol esencial para la búsqueda de la comprensión en las contradicciones sociales que se presentan hoy sobre la diversidad de grupos y la multiplicidad de conductas. Esta situación requiere de un ejercicio reflexivo profundo que se debe lograr en los jóvenes y futuros profesores para una comprensión analítica de la diversidad cultural y heterogeneidad del mundo social (Marín, 2009).

La incorporación de un enfoque de la educación intercultural en la FID habla del reconocimiento e internalización de un saber del otro, que no necesariamente nace y responde a los cánones occidentales, pero que posee para determinados grupos un estatus de conocimiento válido. Por esta razón el enfoque intercultural en educación permite la confrontación de saberes y conocimientos entre personas pertenecientes a esferas culturales diferentes lo que permite alcanzar un nuevo saber sobre la base de un diálogo entre culturas. La apuesta para los espacios fronterizos es una educación que aspira a formar sujetos capaces de descubrir las diferencias, reconocerlas y comprenderlas desde un enfoque relacional de las culturas (Quilaqueo 2007; Quilaqueo y Quintriqueo, 2008).

En suma, el desarrollo de una mirada educativa intercultural fronteriza que permee la FID implica valorar la identidad, la cultura, los procesos, relaciones que se entablan (con distinto nivel y valor) entre los grupos, los contenidos y finalidades educativas de los 'otros' para la formación de personal (Quilaqueo y Quintriqueo 2008; Quilaqueo, Quintriqueo, y Riquelme 2016; Quintriqueo y McGinity, 2009). Asimismo, implica la construcción de conocimientos y competencias dialógicas entre el saber sociocultural de los distintos grupos que ocupan el espacio fronterizo, desde el saber aymara, hasta las costumbres diversas de los migrantes que han sido acogidas en la región, junto al saber occidental que se entrega en el medio escolar formal. De esta manera, la propuesta académica profesional de la formación docente en educación intercultural se fundamenta en el marco de relaciones interétnicas e interculturales (Quilaqueo y Quintriqueo, 2008), caracterizadas por el contacto histórico entre diversos grupos que ha poblado la Región de Arica y Parinacota en el norte de Chile. En este sentido, la FID, respecto a la atención a la diversidad cultural en la escuela se 
debe iniciar con la elaboración de herramientas teórico-metodológicas que nos ayuden a construir una propuesta de educación intercultural para que los profesores sean conscientes de los estereotipos en la enseñanza y de las consecuencias de estos factores sociales y así tengan habilidades y competencias interculturales para lidiar con la diversidad en la sala de clases y evitar procesos de discriminación (Manzi y García, 2016).

Finalmente, una visión crítica de la FID en contextos multiculturales fronterizos lleva a los futuros docentes a descartar de lleno esa relación intercultural entre dos grupos que, por una implementación forzosa de la educación y perspectiva intercultural, se manifiesta en una abierta jerarquía (lo nacional y lo extranjero/cultura nacional y cultura de pueblos originarios, por ejemplo). Si lo anterior es lo que prima, seguro que se generará una interrelación sin comunicación y excluyente que derivará en estereotipos y prejuicios.

\section{LA FORMACIÓN INICIAL DE LOS DOCENTES DE LAS ESCUELAS BÁSICAS DE ARICA Y PARINACOTA}

A continuación, se exponen los resultados obtenidos de la aplicación de las entrevistas sobre la realidad del FID en Escuelas Básicas de la región fronteriza de Arica y Parinacota. Para la realización de las entrevistas sobre la realidad del FID se diseñó un instrumento especifico, una entrevista en profundidad semiestructurada, que agrupó cuatro categorías de análisis y observación con el fin de obtener información de alto contenido y que aportara diferentes perspectivas de análisis de parte de los informantes y permitiera capturar datos más relevantes asociados al objeto de estudio.

Como la información recopilada fue específicamente cualitativa, esto implicó que los datos de la muestra no fueran significativos en cuanto a la cantidad y extensión de ciertas características poblacionales; sino que la relevancia la posee la reconstrucción de las vivencias y sentidos asociados a ciertas instancias microsociales. Por lo tanto, la representatividad de estas no radica en su cantidad sino en las categorías definidas. De este modo, la selección y número de los individuos que participaron en la investigación y que formaron parte de la muestra que tuvo un carácter intencional, estuvo constituida por profesores en ejercicio, lo que fue asociado a los establecimientos educacionales públicos de enseñanza básica con alta presencia de estudiantes extranjeros y de pueblos originarios, con el fin de analizar las problemáticas y oportunidades a las que se enfrentan los profesores en contextos de diversidad. Finalmente, el resultado del ejercicio mencionado fue la identificación de nueve (9) escuelas, lo que permitió entrevistar a 16 profesores. 
Tabla 1.

\begin{tabular}{|c|l|}
\hline $\mathrm{N}^{\circ}$ & \multicolumn{1}{|c|}{ Nombre Escuela } \\
\hline $\mathbf{1}$ & Escuela América \\
\hline $\mathbf{2}$ & Escuela Comandante Juan José de San Martín \\
\hline $\mathbf{3}$ & Escuela Pampa Algodonal \\
\hline $\mathbf{4}$ & Liceo Agrícola Abelardo Núñez \\
\hline $\mathbf{5}$ & Escuela Humberto Valenzuela García \\
\hline $\mathbf{6}$ & Escuela Tucapel \\
\hline $\mathbf{7}$ & Escuela Darío Salas \\
\hline $\mathbf{8}$ & Escuela Manuel Baquedano \\
\hline $\mathbf{9}$ & Escuela España \\
\hline
\end{tabular}

Fuente. Elaboración propia.

\subsection{FID E INTERCULTURALIDAD}

El análisis de la información proporcionada por los profesores entrevistados da cuenta de que durante los años de pregrado la perspectiva intercultural en educación se encuentra ausente o asociada, simplemente, a una visión del ámbito rural a la educación intercultural bilingüe (EIB) que aún marca los espacios urbanos de la comuna de Arica. Recordemos, además, que hoy el currículo incluye una asignatura de lengua y cultura indígenas de forma obligatoria en los establecimientos que poseen más del $20 \%$ de estudiantes indígenas, lo que incide con que la noción de educación intercultural se confunda.

La visión que se transmitía de educación intercultural en la etapa de formación inicial se refleja de la siguiente manera:

...cuando se hablaba de interculturalidad se hablaba de las diferentes formas de educación en establecimientos rurales, pero más que nada desde la perspectiva donde estaban ubicados estos colegios y que existían más que nada una relación unidocente con los alumnos, es decir un solo profesor que hacía de todo en el colegio... en verdad que recuerde no se entregó nada más en relación con este tema (E1).

Otra aseveración vinculada a la FID y educación intercultural relevante es la connotación cercana a lo negativo que atribuyen los profesores entrevistados a la alta carga de contenidos teóricos y no necesariamente relacionados a los temas de diversidad cultural, multiculturalidad o educación intercultural. Simplemente en sus años de FID esperaron más contenidos prácticos que teóricos, puesto que "la teoría" no los preparó para la realidad de sus establecimientos multiculturales. 
En la universidad se pasan muchos contenidos que son teóricos y que al final muchos ni siquiera son aplicables en la realidad, la verdad es que uno se hace profesor en la práctica y no solo desde el punto de vista de trabajo con extranjeros o aymaras que en términos de trabajo con alumnos fue nula, sino en general, solo teoría (E8).

Por otra parte, se rescata la metodología y didáctica entregada que, sin embargo, no incluía aspectos interculturales.

No. La universidad es solo un lugar en donde se nos entrega mucho contenido que muchas veces dista de la realidad y lo poco que se hace con relación a herramientas metodológicas lo hizo (...), pero de educación intercultural o interculturalidad, absolutamente nada (E6).

Sin embargo, se aprecia una contradicción, en su discurso al notar que lo intercultural es relacionado con el mundo andino e indígena a pesar de haber recibido una carga teórica abundante al respecto.

...el profesor (...) y más que nada se hablaba como, solamente de la cultura aymara, como ellos veían su perspectiva de vida, algunas plantas medicinales, cosas, así como temáticas, pero no en profundidad, no como la podemos aplicar (E7).

En el discurso de los docentes se da una correlación positiva cuando indican que lo intercultural en la FID se asoció y vinculó a la cultura y folklore de las poblaciones andinas, llámese fiestas patronales de los distintos pueblos del interior de la región, del calendario agrícola o su ritualidad asociada; o festividades típicas locales como la vendimia, la limpia de canales, cultivo de papa, entre otros.

... un semestre, hicimos salidas a terreno, fuimos al valle de Azapa, hicimos entrevistas, fuimos a Codpa también ese año justo fue el de la Kalapurka más grande, hicimos ese viaje y el profesor tocaba siempre esa temática... (E2).

No, pero había un ramo, o no sé qué, pero no era un ramo así como un $100 \%$ integrado enfocado a las diferentes culturas, se mostraron como las diferentes fiestas y actividades que se hacían en los pueblos de acá de la región (E5).

Esto, forma parte de la manera aun enquistada de apreciar la educación formal cuando trata temas sobre diversidad cultural y, en otras ocasiones, a la educación intercultural bilingüe en una región fronteriza con población originaria que fue foco de la política educativa del EIB. Se genera por lo tanto la distorsión: educación intercultural es igual a educación para la población aymara.

\subsection{NECESIDADES FORMATIVAS}

Una práctica educativa que integre la perspectiva intercultural resulta clave no solo para el logro de aprendizajes significativos en sus estudiantes, sino también para el desarrollo de valores y principios acorde a la diversidad que el espacio fronterizo de la región presenta. De esta forma se indagó en el nivel de preparación de los profesores/as para desempeñarse 
en estas escuelas y, a su vez, qué posibles requerimientos realizan a su proceso de FID o buscan en su Formación Continua. Así algunos profesores manifestaron que improvisaban al momento de aplicar y desarrollar estrategias y trabajos de orden intercultural, basados en lo que ellos entienden y creen que es lo más apropiado, sin existir una capacitación técnica y manejo conceptual de cómo abordar un espacio culturalmente diverso. Lo anterior se aprecia de la siguiente forma:

Totalmente, lo que uno usa es como una invención una especie de Frankenstein de experiencias adquiridas. Sí, claro que, de manera media artesanal, al no haber tenido nunca una referencia real de estas prácticas (E6).

Sí, totalmente, muchas veces los profesores en la práctica debemos improvisar frente a situaciones que van apareciendo ahi en el momento y ahí te das cuenta de que no fuiste bien preparado y que mucho que lo que te enseñaron en la U era solo teoría que se podía practicar muy poco en la realidad (E5).

No, solo a la diversidad de habilidades. Donde logro aplicar algunas cosas que nos enseñan, pero más que nada tiene que ver con la motivación que tengo por trabajar como profesora (E8).

Otros indican que logran salvar situaciones de forma improvisadas o gracias a su creatividad.

Todo lo que he implementado ha sido así como chispazos, pero todavía me falta montones. Todo lo que he hecho es de corazón, de aceptación, de cariño por estos chatitos, veo como los saco adelante, como les saco personalidad, sobre todo a las niñitas, o sea, niñas vamos empodérense, ustedes pueden, eso es solo por amor al arte (E4).

Totalmente, ya que, si bien en la universidad tuvimos temáticas referidas a didáctica y algo de evaluación, no estaban asociadas ni creadas en torno a temas de tipo intercultural (E9).

Entonces este tipo de afirmaciones que contienen palabras claves en el discurso, como improvisación, motivación, ausencia de formación en la temática, vinculación de lo intercultural a lo étnico (aymara), acciones de autoformación y vocación es reiterativo.

Se da, por tanto, una clara ausencia del desarrollo de la perspectiva intercultural práctica en los años de formación, además de existir una disociación que no es acometida por los académicos en cuanto articulación entre teoría y práctica que redunda en la ausencia del desarrollo de competencias interculturales.

Como no tuvimos ese tipo de formación, casi todo lo que aplico son cosas que he ido aprendiendo en mi actual trabajo como profesora (E13).

Me siento preparado debido a mi formación personal no académica, no así por mi formación académica, donde no se nos preparó interculturalmente (E11).

\subsubsection{PERSPECTIVA INTERCULTURAL EN EDUCACIÓN}

La empatía, la educación en valores, la idea de desarrollar la tolerancia ante la diversidad y la inclusión en sus estudiantes son las fortalezas que poseen los profesores/as que formaron 
parte de esta investigación. Ellos, día a día, en su jornada cotidiana están inmersos en un contexto de diversidad cultural que los pone a prueba a cada momento. En todo instante existen situaciones que esperan una respuesta apropiada a ese contexto en particular. No es una tarea sencilla pues sino se posee la experticia y bagaje académico afianzado en una sólida FID o Formación continua concurrente y pertinente, las respuestas no serán las más apropiadas, aunque tengan la intención más positiva.

Sin embargo, la experiencia práctica otorga un saber atingente a estos profesores/as. Esto se demuestra cuando describen situaciones de conflicto o posible discriminación.

A ver, la idea es que, si se detecta una actitud así, hay que inmediatamente corregir y hacer sentido a lo que el colegio postula de aceptar y recibir de manera cordial a los extranjeros y compañeros de etnias, de hecho, en el colegio hay un equipo de convivencia escolar que muchas veces interviene en situaciones de conflicto (E16).

Cuando observo actitudes negativas entre alumnos, referentes a la nacionalidad $u$ origen étnico, les llamó la atención y les trato de explicar mediante ejemplos o alguna actividad que les permita adquirir la empatía necesaria para contrarrestar este tipo de actitudes (E11).

Se muestra la inclusión, dado que los mismos niños son capaces de reconocer a sus pares como un igual, de forma constante reciben una charla por parte del orientador para este y otros temas de importancia para el buen clima estudiantil (E14).

Existen situaciones en las cuales resulta válido preguntarse, ¿desde dónde recupero la experiencia para enfrentarme a esta situación? ¿Desde mi FID o la formación continua me la entregará o debo esperar que la experiencia con el pasar de los años me indique el camino? Estas interrogantes surgen a propósito de los siguientes argumentos emitidos por los docentes.

A veces yo he notado en curso como en $7^{\circ}$ y $8^{\circ}$ básico que existe una negación a autoidentificarse con alguna de las etnias presentes en la región, tal vez producto de la sociedad actual, que los lleva a ser más occidentalizados, lo que les aleja de su propia cultura (E15).

Sin embargo, existe un problema psicopedagógico, debido a que nuestros alumnos están expuestos a pesticidas o agroquímicos a edad temprana lo que a nivel neurológico provoca daño para el aprendizaje (E12).

Aun así, creo que nos encontramos en presencia de una postura y sentido positivo de parte de los profesores/as frente a las temáticas vinculadas a la multiculturalidad, interculturalidad y educación intercultural. Esto es algo relevante si en el futuro se pretende forjar una pedagogía intercultural con carácter fronterizo.

Creo que al momento de tomar la interculturalidad como algo natural positivo etc. Se está desarrollando una práctica intercultural, sin embargo, no es la única, y no es suficiente con solo eso de hecho es como lo mínimo (E13).

Sí, trato de hacer siempre lo que involucre alguna temática que sea diversa, trato de meter siempre que dramaticen, que investiguen, que hagan exposiciones, sobre lo que están hablando, sobre el tema. Si hablamos por ejemplo de los pueblos originarios que hagan dramatizaciones, que busquemos lecturas. Eso hago yo (E2). 
Estudios Pedagógicos XLV, $\mathrm{N}^{\circ}$ 2: 83-99, 2019

FORMACIÓN INICIAL DOCENTE Y ESPACIOS FRONTERIZOS. PROFESORES EN AULAS CULTURALMENTE DIVERSAS. REGIÓN DE ARICA Y PARINACOTA

La notoriedad del vínculo del ejercicio profesional, interculturalidad y comunidad estudiantil de origen aymara es innegable. Todos los entrevistados, sin excepción, manifestaron la alta presencia de estudiantes aymaras y la impronta cultural andina en los contextos donde se encuentran insertos los establecimientos.

Los profesores/as buscan estrategias y formas de establecer una interrelación positiva entre la enseñanza, el rol del EE y esta cultura en particular. Se debe sumar a ello el resto de los estudiantes, en riesgo social, con NEE y extranjeros. Entonces es perfectamente comprensible que se noten carencias o falta de herramientas para abordar esta diversidad, todavía más cuando se debe estar preocupado de rendir en los estándares nacionales y entregar el currículum nacional establecido por el MINEDUC. Por ello no todo será atención a la diversidad. Por esto también, han de ser profesionales de la educación habilidosos para lograr un equilibrio entre todos estos mundos y en este contexto nuevamente la función de la FID es clave.

Se hace, pero no por etnia sino por sus capacidades, se trata de nivelar a todos por igual y no hacemos la prueba en quechua ni en aymara, porque no hay niños que hablen el 100\% la lengua. En síntesis, se aplica la misma prueba, se evalúa lo que se enseñó del mismo contenido. La diferencia se hace por capacidades (E3).

(los aymaras) solo se diferencian en aprendizaje en sus prácticas, algunos son más concretos, otros más lentos. Se pasan los contenidos tal cual, y se hacen adaptaciones para los niños del proyecto de integración, solo a ellos, o sea no se hace nada más que eso (E14).

\subsubsection{ESTRATEGIAS PEDAGÓGICAS}

Los profesores/as entrevistados fueron consultados sobre las estrategias que utilizan o se aprecian en su escuela para el desarrollo de una educación intercultural. Lo anterior a fin de apreciar qué tan cerca o lejos se está para hablar de una práctica pedagógica intercultural. El repertorio es amplio y diverso, con matices y críticas a sus establecimientos destacando el esfuerzo propio por desarrollar estrategias para la inclusión y la iniciativa personal que ello implica. Sin embargo, se evidencia que durante los años de pregrado la perspectiva educativa intercultural nuevamente se encuentra asociada, simplemente, a una visión del ámbito rural, al desempeño unidocente en contextos rurales y a la educación intercultural bilingüe que aun marca los espacios urbanos de la comuna de Arica.

Con instrumentos musicales que son de origen de distintos países, además de como dije anteriormente recatando la música y danza que mis propios alumnos traen y comparten con sus compañeros. En música siempre hay temáticas y contenidos con relación a cultura tradicional y folklore.

Lo que yo hago es transversal y en mi colegio hay una profesora de aymara, por ejemplo, el Machaq Mara se celebra en grande, yo no siendo de la raza del paisaneo de ellos, trato de involucrarme también, me visto llevo mi aguayo, hemos hecho paguas, trato de saludar. Sumauru Yatichiri...(E9).

Las evaluaciones son diversas, si bien hoy en día el sistema está por evaluar los estudiantes por la famosa prueba SIMCE, las evaluaciones son de alternativas, pero mayormente por una cosa de condicionar llamémosla así, cruelmente de condicionar 
al estudiante para un formato de prueba que aplica el estado, son pruebas más de alternativas las pruebas sumativas (E10).

Se extraña una mirada más reflexiva y crítica de parte de los profesores sobre lo que implica enseñar en diversidad, más bien se deslinda responsabilidad en la institución, que da cuenta de la visión jerárquica del establecimiento y que no se sienten parte de cambios como agentes para el mismo.

\section{CONCLUSIONES}

La Región de Arica y Parinacota se ha evidenciado como un espacio donde cada establecimiento de educación pública demuestra ser un lugar culturalmente diverso producto de la dinámica propia de un territorio en el que convergen una tradición cultural propia de un pueblo originario y las dinámicas de los flujos migratorios característicos de un territorio fronterizo. La multiplicidad de realidades de las escuelas regionales, conocidas a través de los/as profesores/as entrevistados/as, permitió un acercamiento a la formación inicial de los/as profesionales de la educación y los problemas que presenta el ejercicio y práctica docente en contextos de diversidad cultural. Las experiencias son tan heterogéneas y diversas como el alumnado de las escuelas donde se desempeñan los/as profesores/as.

Ahora bien, es posible concluir que la FID recibida por los/as docentes no involucró de manera intencionada y sistemática un enfoque en educación intercultural, ya sea de forma teórica como práctica. La forma superficial del tratamiento de los temas asociados a la diversidad cultural en el ámbito de la educación formal y la educación intercultural como modelo de gestión de la diversidad cultural, durante la formación inicial, hace que los/as profesores/as no se sientan completamente capacitados aún para desempeñar y desarrollar una práctica pedagógica en un establecimiento culturalmente diverso. Sin embargo, esta situación no indica que su desempeño sea inadecuado para este contexto. Por el contrario, el trabajo realizado en sus años de ejercicio los ha dotado de una experiencia que les permite hacer frente al desafío que implica el desarrollar una práctica educativa en escuelas culturalmente diversas y les permite aportar con ello al desarrollo y progreso de una incipiente educación intercultural.

Asimismo, la formación recibida durante los años posteriores al término de su FID se transformó en un espacio débilmente propicio para la incorporación y el desarrollo de andamiajes teóricos y conceptuales de la interculturalidad y la educación intercultural. Básicamente, los procesos de capacitación estuvieron determinados por la adquisición de nuevas capacidades y habilidades con miras a fortalecer su práctica educativa y mejorar los resultados y rendimiento en determinadas materias.

La ausencia de la perspectiva intercultural y por derivación de una formación en educación intercultural en el perfil profesional demuestra el largo camino que todavía se debe recorrer para incorporar esta discusión tan relevante al interior de la FID en una región fronteriza que permita, en definitiva, desplegar prácticas interculturales. De esta forma la incorporación de actividades, estrategias y técnicas de enseñanza y evaluación que involucra la educación intercultural o que se puedan denominar interculturales, necesaria para estos establecimientos, es producto de una motivación e iniciativa profesional personal y del contexto que obliga al/la docente a generar actividades 
y estrategias con claros elementos locales, intuitivas y autodidactas para el logro de sus objetivos de aprendizaje. Con todo, es posible afirmar que existe un desarrollo y construcción de un conocimiento y saber docente situacional y contextual en el que se aprecia el brillo tenue de la interculturalidad. Dicho esto, las prácticas pedagógicas están fundadas en saberes que no necesariamente están basados en los principios de la educación intercultural y no nacen a partir de un proceso formativo, sino del contexto y el ejercicio educativo diario.

Por otra parte, no fue posible identificar un esfuerzo integral por parte de los distintos agentes educativos de la región para el desarrollo de este tipo de educación. De ahí que solo existan esfuerzos intuitivos que, a través de actividades supuestamente interculturales, los/as profesores/as visualizan el logro de una educación inclusiva e intercultural pertinente a su establecimiento. En consecuencia, la creencia de que la educación intercultural es el rescate de las tradiciones, la mantención de la lengua, la utilización de ritmos folclóricos, la utilización de instrumentos musicales diversos, el uso de palabras en aymara, es parte de la evidencia que demuestra que el proceso de FID vivido no tenía como eje articulador el tratamiento de forma sistemática la diversidad cultural, sus problemáticas, discusiones conceptuales y teóricas, junto a sus implicancias en el quehacer pedagógico y educativo.

La práctica educativa al no estar determinada por los principios de la educación intercultural hace posible la emergencia y generación de dispositivos discursivos de exclusión o discriminatorios poco inclusivos. De esta forma, el/la profesor/a que no recibió una formación en educación intercultural, y si sumamos que no es un pedagogo reflexivo y crítico de su ejercicio, no logrará notar la carga de prejuicios o estereotipos que puede llegar a contener su discurso educativo.

Respecto de las necesidades o carencias formativas de los/as profesores/as en ejercicio en espacios multiculturales fronterizos, un alto porcentaje manifestó haber enfrentado un choque en el aula producido por la diversidad cultural con la que se encontraron y para la que no se sintieron preparados. De esto se concluye la ausencia del desarrollo de competencias de carácter intercultural o de atención a la diversidad durante su FID, las que habrían brindado, en parte, una guía para iniciar la tarea de enseñar de forma diferenciada. Las situaciones fueron salvadas cuando recurrieron a su ingenio para diseñar estrategias didácticas que pudieran involucrar la diversidad cultural a la que siguieron enfrentándose en sus primeros años de ejercicio una vez egresados de la universidad.

La visión que poseen los/as profesores/as sobre su FID para el desempeño en estos contextos contiene elementos críticos sobre su proceso formativo que se resumen en que el programa académico cursado tuvo un carácter más teórico que práctico. Dichos aspectos teóricos no necesariamente estuvieron inscritos en el eje o enfoque de la educación intercultural, sino más bien se vinculó a todo lo referente a la tradición cultural aymara. Esta situación trasunta a la práctica educativa, que quedó en evidencia, cuando los/as profesores/as hablaban de la interculturalidad como un auténtico sinónimo de lo aymara. Finalizando, la formación inicial docente recibida por los/as profesores/ as de estos contextos debe ser, en la óptica de la educación intercultural, una formación reflexiva y crítica de la realidad sociocultural local y regional, pues aquello permitirá que el docente involucre la diversidad presente en la región fronteriza de Arica y Parinacota a su práctica educativa para hacerla intercultural. 


\section{REFERENCIAS BIBLIOGRÁFICAS}

Aguado, T. (2009). El enfoque intercultural como metáfora de la diversidad en educación. En T. Aguado y M. del Olmo (Eds.), Educación Intercultural perspectivas y propuestas (pp. 15-29). Madrid: Editorial Universitaria Ramón Areces / UNED.

Alliaud, A. y Vezub, L. (2014) La formación inicial y continua de los docentes en los países del MERCOSUR. Problemas comunes, estructuras y desarrollos diversos. Cuadernos de Investigación Educativa, 5(20), 31-46.

Barrios, Ll. y Palou, B. (2014). Educación intercultural en Chile: la integración del alumnado extranjero en el sistema escolar. Educación y Educadores, 17(3), 405-426.

Becerra, G. (2005). Necesidades de formación docente en la zona de frontera: una exploración inicial. Aldea mundo, 9(17), 33-40.

Celedón, M., Naudon, P. y Carmona, K. (2018). Las nuevas aulas multiculturales en Chile, un diagnóstico. EduGlobal. www.eduglogal.cl. Consultado en octubre de 2018.

Cerón, L., Pérez, M. y Poblete, R. (2017). Percepciones docentes en torno a la presencia de niños y niñas migrantes en escuelas de Santiago: Retos y desafíos para la inclusión. Revista latinoamericana de educación inclusiva, 11(2), 233-246.

Díaz, A. (2003). Problemas y perspectivas sociohistóricas en el norte chileno: Análisis sobre la "chilenización" de Tacna y Arica. Revista si somos americanos. Revista de Estudios Transfronterizos, 5(4), 49-81.

Dietz, G. (2003). Multiculturalismo, interculturalidad y educación: una aproximación antropológica. Granada: Fondo de Cultura Económica.

(2005). La interculturalidad entre el empoderamiento de las minorías y la gestión de la diversidad. Puntos de vista. Cuadernos del observatorio de las migraciones y la convivencia intercultural de la ciudad de Madrid, 12(3), 27-44.

. (2013). Multiculturalismo, interculturalidad y diversidad en educación. Una aproximación antropológica. México: Fondo de Cultura Económica.

Dorfsman, M. (2012). La profesión docente en contextos de cambio: el docente global en la sociedad de la información. RED-DUSC. Revista de Educación a Distancia-Docencia Universitaria en la Sociedad del Conocimiento, 6, 1-23. Disponible en: http://www.um.es/ead/reddusc/6/. Consultado en septiembre de 2018.

Enríquez, P. (2007). Formación inicial de los docentes-investigadores en metodología de la investigación. En P. Enríquez, El docente-investigador: Un mapa para explorar un territorio complejo (pp. 87-110). San Luis: Ediciones LAE.

Essomba, M. (2008). 10 ideas clave. La gestión de la diversidad cultural en la escuela. Barcelona: Graó.

García, J. (2005). Educación intercultural. Análisis y propuestas. Revista de Educación, 336, 89-109.

Geeregat, O., Vásquez, O. y Fierro, J. (2012). Procesos de formación inicial docente en contextos multiculturales: inclusión y exclusión. Estudios Pedagógicos, XXXVIII(1), 345-351.

Guizardi, M. y Garcés H. (2012). Mujeres peruanas en las regiones del Norte de Chile: apuntes preliminares para la investigación. Estudios Atacameños, (44), 5-34.

Hirmas, C. y Cortés, I. (2013). Estado del arte. Investigaciones sobre formación práctica en Chile: tensiones y desafíos. Santiago: Organización de Estados Iberoamericanos para la Educación, la Ciencia y la Cultura.

Joiko, S. y Vásquez, A., (2016). Acceso y elección escolar de familias migrantes en Chile: No tuve problemas porque la escuela es abierta, porque acepta muchas nacionalidades. Calidad en la Educación, (45), 132-173.

Manzi, J. y García, M. (2016). Abriendo las puertas del aula. Transformaciones de las prácticas docentes. Santiago: Ediciones UC.

Marín, J. (2009). La perspectiva intercultural como base para imaginar una educación democrática 
Estudios Pedagógicos XLV, N 2: 83-99, 2019

FORMACIÓN INICIAL DOCENTE Y ESPACIOS FRONTERIZOS. PROFESORES EN AULAS CULTURALMENTE

DIVERSAS. REGIÓN DE ARICA Y PARINACOTA

para los pueblos autóctonos y para la sociedad multicultural en América Latina. Sociedad y Discurso, 15(1), 54-89.

MINEDUC (2015). Estudios sobre Formación Inicial Docente (FID) en Chile. Ministerio de Educación de Chile.

Mondaca, C. y Gajardo, Y. (2015). Interculturalidad, Migrantes y Educación (Editorial). Diálogo Andino, (47), 3-6.

Mondaca, C., Gajardo, Y. y Sánchez, E. (2016). Historia, memoria y ciudadanía intercultural. El reto del siglo XXI para las aulas de la región de Arica y Parinacota, norte de Chile. Interciencia, 39(7), 524-530.

Mondaca, C., Rivera, P. y Gajardo, Y. (2014). Educación parvularia e inclusión en el norte de Chile: formando pequeños chilenos en las aulas de Tarapacá. Revista Alpha, (39), 251-266.

Morales, C. (2015). Nociones y principios de la educación intercultural presentes en prácticas pedagógicas realizadas en contexto de interacción juvenil rural de la región metropolitana (Chile). Diálogo Andino, (47), 59-70.

Norambuena, C. (2002). Migraciones limítrofes. Chile 1865-1960. En H. Asdrúbal (Ed.), Historia de las Migraciones Limítrofes en el Cono Sur de América (Vol. 2) (pp. 25-92). México: Instituto Panamericano de Geografía e Historia.

. (2004). Migraciones transfronterizas en el cono sur de América. En Informe sobre el Estado de la integración (pp. 143-154). Instituto Internacional de Integración. La Paz: Convenio Andrés Bello.

Pries, L. (1999a). La migración internacional en tiempos de globalización: varios lugares a la vez. Nueva Sociedad, (164), 56-68.

(1999b). Una nueva cara de la migración globalizada: el surgimiento de nuevos espacios sociales transnacionales y plurilocales. Seminario de globalización y territorio. Red interamericana de investigadores sobre globalización y territorio. Toluca, México.

Quilaqueo, D. (2007). Saberes y conocimientos indígenas en la formación de profesores de educación intercultural. Educar, (29), 223-239.

Quilaqueo, D. y Quintriqueo, S. (2008). Formación docente en educación intercultural para contexto mapuche en Chile. Cuadernos Interculturales, 6(10), 91-110.

Quintriqueo, S. y McGinity, M. (2009). Implicancias de un modelo curricular monocultural en la construcción de la identidad sociocultural de alumnos/as mapuches de la IX región de La Araucanía, Chile. Estudios Pedagógicos (Valdivia), 35(2), 173-188.

Quilaqueo, D., Quintriqueo, S. y Riquelme, E. (2016). Identidad Profesional Docente: Práctica Pedagógica en Contexto Mapuche. Estudios pedagógicos, 42(2), 269-284.

Ruffinelli, A. (2016). Ley de desarrollo profesional docente en Chile: de la precarización sistemática a los logros, avances y desafíos pendientes para la profesionalización. Estudios pedagógicos, 42(4), 261-279.

Sanhueza, V., Friz, M. y Quintriqueo, S. (2014). Estudio exploratorio sobre las actitudes y comportamiento del profesorado de Chile en contextos de escolarización de alumnado inmigrante REDIE. Revista Electrónica de Investigación Educativa, 16(3), 14-162.

Stefoni, C., Stang, F. y Riedemann, A. (2016). Educación e interculturalidad en Chile: un marco para el análisis. Estudios Internacionales, 48(185), 153-182.

Tapia, M. (2015). Frontera, movilidad y circulación reciente de peruanos y bolivianos en el norte de Chile. Estudios Atacameños. Arqueología y Antropología Surandinas, (50), 195-213.

UNESCO (2017). Competencias Interculturales. Marco conceptual y operativo. Universidad Nacional de Colombia, Cátedra UNESCO -Diálogo intercultural, Competencias Interculturales: marco conceptual y operativo.

Vaillant, D. (2013). Formación inicial del profesorado en América Latina: dilemas centrales y perspectivas. Revista Española de Educación Comparada, (22), 185-206.

Valenzuela, J., Muñoz, C. y Marfull, M. (2018). Perfiles motivacionales durante la formación inicial 
docente. Profesorado. Revista. De currículum y formación del profesorado, 22(1), 325-346.

Vezub, L. (2016). Los saberes docentes en la formación inicial. La perspectiva de los formadores.

Pensamiento Educativo. Revista de Investigación Educacional Latinoamericana, 53(1), 1-14.

Zapata, R. (2013). Formación del futuro profesorado en diversidad cultural: Estudio de un caso en el contexto universitario chileno. Akadèmeia, 2(2), s.p.

(2014). Diversidad cultural en la formación del futuro profesorado en América latina. Necesidades y perspectivas. Revista interuniversitaria de formación del profesorado, 17(2), 219-234. 
